\title{
Optimized Geometry, Charge Distributions, Frontier Molecular Orbital Analysis and NLO Efficiency of BIS (4-Methoxyanilinium) Adipate by Quantum Chemical Calculations
}

\author{
V. Siva, A. Shameem, A. Murugan, S. Athimoolam, S. Asath Bahadur
}

\begin{abstract}
In this work, optimized molecular geometry, vibrational and electronic properties of Bis(4-methoxylanilinium) adipate (4MAA) was obtained by Quantum chemical analysis using 6-311++G(d,p) basis sets. The experimental and computation vibrational spectra have been discussed. Experimental spectra of the $4 M A A$ were recorded in the region $4000-400 \mathrm{~cm}^{-1}$. The experimental and computational assignments were found to be in good agreement. FMO energies showed that charge transfer had occurred within the molecule. Mulliken charge distribution of the present compound was studied systematically. Furthermore, the hyperpolarizability calculations were analyzed.
\end{abstract} $N L O$

Keywords: Organic, molecular geometry, HOMO-LUMO and

\section{INTRODUCTION}

$S_{\text {chiff based compounds are generally considered due to }}$ their importance in several electro-optical and other technological applications. $\pi$-electron conjugation with aromatic structure is essential to exhibit the nonlinear optical (NLO) properties and on this basis the aniline is consider as one the systems for NLO applications.

Revised Manuscript Received on December 15, 2019.

V. Siva, Department of Physics, Kalasalingam Academy of Research and Education, Krishnankoil-626 126, India, and Condensed Matter Physics Laboratory, International Research Centre, Kalasalingam Academy of Research and Education, Krishnankoil-626 126, India. Email: vsiva33.physics@gmail.com

A. Shameem, Department of Physics, Kalasalingam Academy of Research and Education, Krishnankoil-626 126, India, and Condensed Matter Physics Laboratory, International Research Centre, Kalasalingam Academy of Research and Education, Krishnankoil-626 126, India. Email: shameemirfu@gmail.com.

A. Murugan, Department of Physics, Kalasalingam Academy of Matter Physics Laboratory, International Research Centre, Kalasalingam Academy of Research and Education, Krishnankoil-626 126, India. Email:murugan027@gmail.com.

S. Athimoolam, Department of Physics, University College of Engineering Nagercoil, Anna University, Nagercoil- 629 004, India, Email:athi81s@yahoo.co.in.

S. Asath Bahadur*, Department of Physics, Kalasalingam Academy of Research and Education, Krishnankoil-626 126, India, and Condensed Matter Physics Laboratory, International Research Centre, Kalasalingam Academy of Research and Education, Krishnankoil-626 126, India. Email: s_a_bahadur@yahoo.co.in. Research and Education, Krishnankoil-626 126, India, and Condensed

This statement is substantiated by many theoretical and experimental works [1,2]. Aniline based compounds have been widely used as starting materials in a vast amount of pharmaceuticals, optoelectronic devices, dyes and other technical applications [3-5]. Charge transfer based organic molecules have been established in past decades to possess more higher order NLO properties compared to the more conventional inorganic compounds. The present work is focused on the molecular structure optimization by Quantum chemical analysis using $6-311++\mathrm{G}(\mathrm{d}, \mathrm{p})$ basis sets. The computational calculations using HF and DFT methods have been carried out. Nonlinear optical parameters such as polarizability, first and second order hyperpolarizabilities were calculated. FMOs analysis and charge distribution of the title compound also explained in detail.

\section{RESULTS AND DISCUSSION}

\section{A. Molecular geometry}

The planarity of the structure is confirmed from the torsion angles and these planar molecules are oriented with an angle of 71.03(4) $\AA$ to each other. Protonation on the N-site of the cation is confirmed from elongated $\mathrm{C}-\mathrm{N}$ bond distance [1.456(3) $\AA$ ]. Most of the optimized structural parameters are slightly higher than the single crystal XRD values [6], due to the computational calculations belong to isolated molecules gases state (Table I). Optimized Structure for the title compound given in Fig. 1 and Fig. 2.
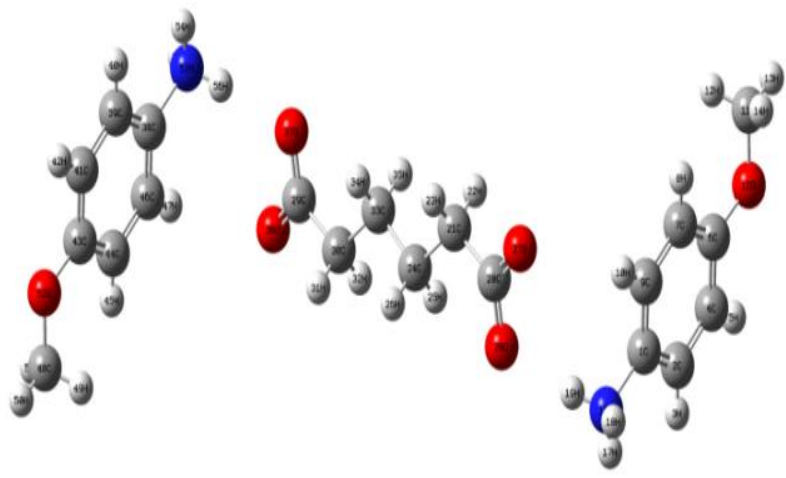

Fig.1. Optimized Structure for DFT/6-311++G(d,p). 


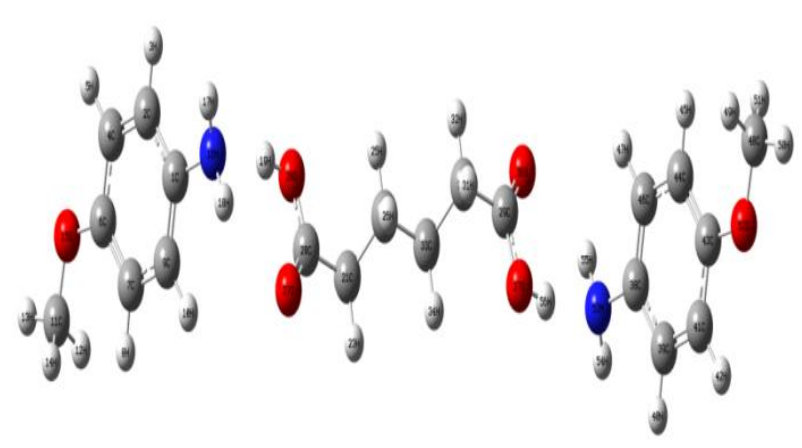

Fig. 2. Optimized Structure for HF/6-311++G(d,p)

\section{B. Mulliken charge distribution analysis}

The histogram of atomic charges of 4MAA was shown in Fig. 3. The hydrogen atom (H56) in amine group of 4MAA was more electropositive (0.684e in DFT and $0.408 e$ in HF), this hydrogen atom was attached with electronegative atom (N53). Also, oxygen atom has more electro negativity (027) $(-0.849 e$ in DFT and $-0.531 e$ in HF), this atom was enclosed by one electronegative oxygen atom $(\mathrm{O} 28)$ and one carbon atom (C20). The oxygen atoms which are involved in intermolecular hydrogen bending interactions between the anions show electronegative charge values.

\section{HOMO-LUMO analysis}

FMO analysis of 4MAA are calculated with Gaussian 09W[7] program to calculate the FMO energy gap. The charge transfer of the molecule is also established by the topology of these orbitals and it is shown in Fig. 5. The calculated FMO energy values and other parameters of 4MAA are given in Table 1. Therefore the lowering in the FMO energy gap obviously explains the concluding charge transfer interactions taking place within the molecule and good candidate for NLO applications.

\section{Vibrational analysis}

The observed and computed FTIR and FT-Raman spectra are shown in Figs. 6 and 7 and the detailed vibrational assignments (Table II) visualized through Gauss view [7] are discussed below.

\section{Vibration of C-H, C-N, C-C and C=O group}

The $\mathrm{C}-\mathrm{H}$ in-plane bending vibration was observed at 1152 $\mathrm{cm}^{-1}$ in IR and well matched with the theoretically counterparts. $\mathrm{C}-\mathrm{H}$ out of plane bending vibration were calculated at 1010, $1049 \mathrm{~cm}^{-1}$ in $\mathrm{HF}$ and $936,953 \mathrm{~cm}^{-1}$ in B3LYP methods. The asymmetric stretching of $\mathrm{COOH}$ group observed at $1950 \mathrm{~cm}^{-1}$ in IR and it is computed at 1959 and $1763 \mathrm{~cm}^{-1}$ in the HF and B3LYP levels respectively. Also, the symmetric stretching of $\mathrm{COOH}$ group is absent in Raman spectrum. The carboxylic group out-plane bending vibrations is computed at 3158 and $2999 \mathrm{~cm}^{-1}$ in the HF and B3LYP levels respectively, but it is absent in experimental IR and Raman spectra. The title compound, 4MAA have the $\mathrm{C}-\mathrm{N}$ and $C=C$ stretching vibrations occurs in the range $1300-1600$ $\mathrm{cm}^{-1}$ owing to literature. The $\mathrm{C}=\mathrm{C}$ stretching mode of vibration is observed at $1525 \mathrm{~cm}^{-1}$ in IR and computed at 1548 in $\mathrm{HF}$ and $1456 \mathrm{~cm}^{-1}$ in DFT/B3LYP methods, respectively. The C-N bending mode is observed at $1552 \mathrm{~cm}^{-1}$ and 1457 $\mathrm{cm}^{-1}$ in HF and DFT/B3LYP methods. This corresponding vibration was absent in experimental spectrum.

\section{Vibration of methyl Group}

The stretching vibrations of methyl group were excepted in the range $3010-2900 \mathrm{~cm}^{-1}$. In the present study, the asymmetric stretching vibrational mode is found at the frequency of $3255 \mathrm{~cm}^{-1}$ in FT-IR spectrum. In $\mathrm{HF}$ and DFT/B3LYP calculation, the $\mathrm{CH}_{3}$ asymmetric stretching vibration is identified at 3279 and $3131 \mathrm{~cm}^{-1}$ respectively whereas in Raman spectrum it is absent. The $\mathrm{CH}_{3}$ scissoring vibration was observed at 1631 and $1506 \mathrm{~cm}^{-1}$ in $\mathrm{HF}$ and DFT/B3LYP methods, respectively. The same vibration was observed around $1635 \mathrm{~cm}^{-1}$ in IR spectrum and absent in Raman spectrum. The $\mathrm{CH}_{3}$ wagging mode at $1593 \mathrm{~cm}^{-1}$ in Raman spectra and agree well with theoretical results at 1587 and $1471 \mathrm{~cm}^{-1}$ in HF and DFT/B3LYP methods respectively. The rocking vibration was observed around 1620 and 1492 $\mathrm{cm}^{-1}$ in $\mathrm{HF}$ and DFT/B3LYP methods, respectively. Experimental spectra are absent for these vibrations. 
Table I: Optimized molecular geometrical parameters for 4MAA

\begin{tabular}{|c|c|c|c|}
\hline Atom Connected & Experimental & HF/ & B3LYP \\
\hline \multicolumn{4}{|c|}{ Bond length $(\AA)$} \\
\hline $\mathrm{C} 1-\mathrm{C} 2$ & $1.380(4)$ & 1.393 & 1.380 \\
\hline $\mathrm{C} 1-\mathrm{C} 9$ & $1.361(4)$ & 1.380 & 1.361 \\
\hline C1-N16 & $1.456(3)$ & 1.418 & 1.456 \\
\hline $\mathrm{C} 2-\mathrm{H} 3$ & 0.93 & 1.077 & 0.930 \\
\hline $\mathrm{C} 1-\mathrm{C} 4$ & $1.376(4)$ & 1.377 & 1.380 \\
\hline $\mathrm{C} 4-\mathrm{H} 5$ & 0.93 & 1.075 & 0.930 \\
\hline C4-C6 & $1.382(4)$ & 1.392 & 1.382 \\
\hline C6-C7 & $1.380(4)$ & 1.382 & 1.380 \\
\hline $\mathrm{C} 11-\mathrm{O} 15$ & $1.404(4)$ & 1.397 & 1.404 \\
\hline $\mathrm{C} 20-\mathrm{C} 21$ & $1.508(3)$ & 1.507 & 1.508 \\
\hline $\mathrm{C} 20-\mathrm{O} 27$ & $1.252(3)$ & 1.191 & 1.252 \\
\hline $\mathrm{C} 20-\mathrm{O} 28$ & $1.260(3)$ & 1.317 & 1.260 \\
\hline $\mathrm{C} 21-\mathrm{H} 22$ & 0.97 & 1.086 & 0.970 \\
\hline $\mathrm{C} 21-\mathrm{H} 23$ & 0.97 & 1.083 & 0.970 \\
\hline $\mathrm{C} 21-\mathrm{C} 24$ & $1.518(4)$ & 1.530 & 1.523 \\
\hline $\mathrm{C} 24-\mathrm{H} 25$ & 0.97 & 1.086 & 0.970 \\
\hline $\mathrm{C} 24-\mathrm{H} 26$ & 0.97 & 1.088 & 0.970 \\
\hline C24-C33 & $1.523(3)$ & 1.528 & 1.518 \\
\hline \multicolumn{4}{|c|}{ Bond Angle $\left(^{\circ}\right)$} \\
\hline $\mathrm{C} 2-\mathrm{C} 1-\mathrm{C} 9$ & $119.9(2)$ & 118.391 & 119.947 \\
\hline $\mathrm{C} 2-\mathrm{C} 1-\mathrm{N} 16$ & $119.8(2)$ & 120.490 & 119.805 \\
\hline C9-C1-N16 & $120.2(2)$ & 121.054 & 120.239 \\
\hline $\mathrm{C} 1-\mathrm{C} 2-\mathrm{H} 3$ & 120.4 & 119.801 & 120.430 \\
\hline $\mathrm{C} 1-\mathrm{C} 2-\mathrm{C} 4$ & $121.2(2)$ & 120.825 & 119.147 \\
\hline C4-C6-C7 & $118.7(3)$ & 118.918 & 118.715 \\
\hline C4-C6-O15 & $122.8(2)$ & 116.090 & 116.030 \\
\hline C7-C9-H10 & 109.4 & 119.175 & 119.475 \\
\hline C6-O15-C11 & $118.2(2)$ & 119.622 & 118.248 \\
\hline C1-N16-H17 & 109.5 & 112.314 & 109.477 \\
\hline C1-N16-H18 & 109.5 & 112.287 & 109.471 \\
\hline \multicolumn{4}{|c|}{ Torsional Angle $\left(^{\circ}\right)$} \\
\hline $\mathrm{H} 25-\mathrm{C} 24-\mathrm{C} 33-\mathrm{C} 30$ & $57.5(3)$ & 58.346 & 59.0529 \\
\hline H25-C24-C33-H34 & $178.2(2)$ & 179.999 & 179.997 \\
\hline $\mathrm{H} 26-\mathrm{C} 24-\mathrm{C} 33-\mathrm{H} 35$ & $179.5(1)$ & 180.000 & 180 \\
\hline $\mathrm{H} 45-\mathrm{C} 44-\mathrm{C} 46-\mathrm{C} 38$ & $178.2(2)$ & 179.920 & 178.777 \\
\hline
\end{tabular}


Optimized Geometry, Charge Distributions, Frontier Molecular Orbital Analysis and NLO Efficiency of BIS (4-Methoxyanilinium) Adipate by Quantum Chemical Calculations

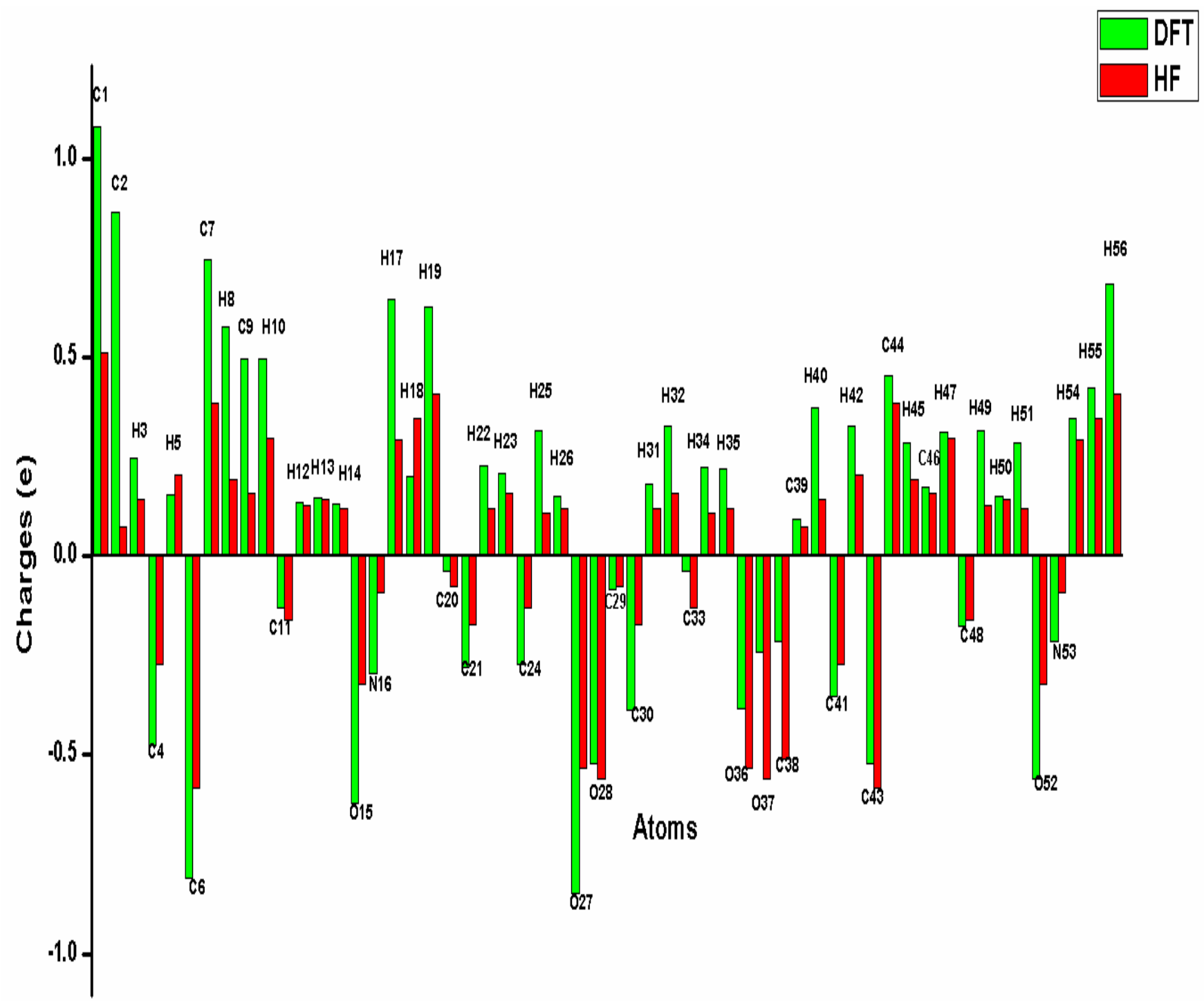

Fig.3. Mulliken charge distributions for 4MAA

(a)

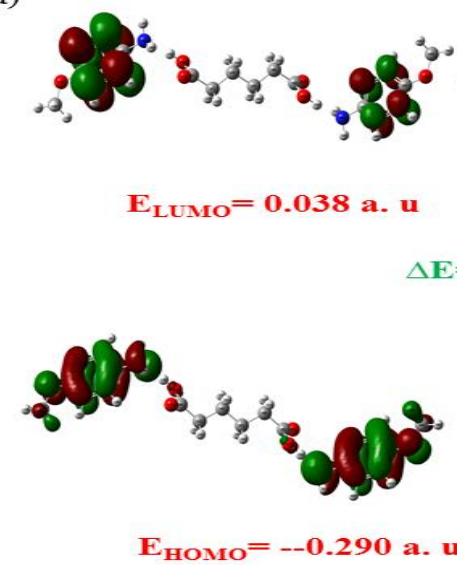

(b)

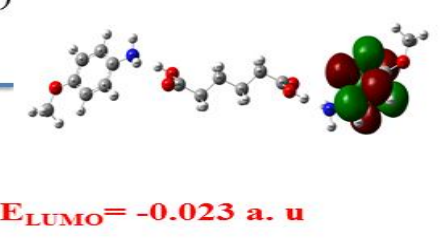

$\Delta \mathbf{E}=0.188$ a. $\mathbf{u}$

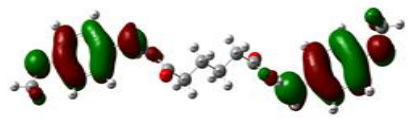

$\mathbf{E}_{\text {номо }}=-0.211$ a. $u$

Fig. 4. Molecular orbital energy diagram for 4MAA

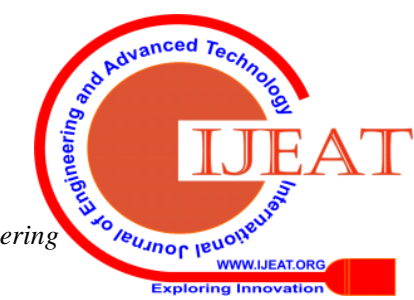


Table II: Calculated energy values of 4MAA

\begin{tabular}{|c|c|c|}
\hline Parameters (au) & $\mathrm{HF}$ & B3LYP \\
\hline $\begin{array}{l}E_{\text {LUMO }} \\
\end{array}$ & 0.038 & -0.023 \\
\hline$\overline{\mathrm{E}_{\text {HOMO }}}$ & -0.29 & -0.211 \\
\hline$\Delta\left(\mathrm{E}_{\mathrm{LUMO}}-\mathrm{E}_{\mathrm{HOMO}}\right)$ & 0.252 & 0.188 \\
\hline Electron Affinity (A) & -0.038 & 0.023 \\
\hline Ionization Potential (I) & 0.291 & 0.211 \\
\hline Chemical Hardness $(\eta)$ & 0.164 & 0.094 \\
\hline Electronegativity $(\chi)$ & 0.126 & 0.117 \\
\hline Chemical Potential $(\mu)$ & -0.126 & -0.117 \\
\hline Electrophilicity Index $(\omega)$ & 0.049 & 0.073 \\
\hline
\end{tabular}

Table III: Vibrational Assignments of 4MAA

\begin{tabular}{|c|c|c|c|c|c|c|c|c|c|}
\hline \multirow{2}{*}{$\begin{array}{c}\text { Mode } \\
\text { Number }\end{array}$} & \multicolumn{2}{|c|}{ Experimental } & \multicolumn{3}{|c|}{$\mathrm{HF} / 6-311++\mathrm{G}(\mathrm{d}, \mathrm{p})$} & \multicolumn{3}{|c|}{ B3LYP/6-311++G(d,p) } & \multirow{2}{*}{ Assignment } \\
\hline & FT-IR & Raman & $v_{\text {cal }}$ & ${ }^{\mathrm{a}} \mathrm{I}^{\mathrm{IR}}$ & ${ }^{b} I^{\text {Raman }}$ & $v_{\mathrm{cal}}$ & ${ }^{\mathrm{a}} \mathrm{I}^{\mathrm{IR}}$ & ${ }^{b} I^{\text {Raman }}$ & \\
\hline 1 & & & 4 & 0.073 & 0.000 & 4 & 0.007 & 1.850 & $\omega\left(\mathrm{CH}_{2}\right.$ of $\left.\mathrm{CH}_{3}\right)$ \\
\hline 2 & & & 5 & 0.009 & 0.000 & 7 & 0.024 & 1.497 & $\omega\left(\mathrm{CH}_{3}\right)$ \\
\hline 3 & & & 8 & 0.000 & 1.784 & 10 & 0.014 & 1.514 & $\gamma(\mathrm{CH})$ \\
\hline 4 & & & 14 & 0.515 & 0.000 & 17 & 0.158 & 2.596 & $\gamma(\mathrm{CH})$ \\
\hline 5 & & & 14 & 0.000 & 1.635 & 23 & 1.561 & 1.472 & $\gamma(\mathrm{CH})$ \\
\hline 6 & & & 25 & 1.369 & 0.000 & 25 & 2.025 & 2.205 & $\gamma(\mathrm{CH})+\omega\left(\mathrm{CH}_{2}\right.$ of $\left.\mathrm{CH}_{3}\right)$ \\
\hline 7 & & & 27 & 0.000 & 5.896 & 32 & 0.385 & 3.210 & $\omega(\mathrm{C}=\mathrm{O})+\gamma(\mathrm{CH})$ \\
\hline 8 & & & 33 & 2.848 & 0.000 & 41 & 0.403 & 2.619 & $\omega(\mathrm{C}=\mathrm{O})+\gamma(\mathrm{CH})$ \\
\hline 9 & & & 40 & 0.000 & 1.742 & 42 & 0.943 & 1.027 & $\gamma(\mathrm{CH})$ \\
\hline 10 & & & 49 & 4.489 & 0.000 & 48 & 1.979 & 1.188 & (C-O) Stretching \\
\hline 11 & & & 52 & 0.000 & 1.578 & 52 & 0.210 & 2.066 & $\gamma(\mathrm{CH})+\omega\left(\mathrm{CH}_{2}\right.$ of $\left.\mathrm{CH}_{3}\right)$ \\
\hline 12 & & & 63 & 0.000 & 1.589 & 74 & 2.773 & 1.080 & $\omega\left(\mathrm{CH}_{3}\right)$ \\
\hline 13 & & & 67 & 8.037 & 0.000 & 80 & 5.642 & 0.660 & $\omega\left(\mathrm{CH}_{3}\right)$ \\
\hline 14 & & & 79 & 0.000 & 1.373 & 88 & 3.396 & 0.630 & $\omega\left(\mathrm{CH}_{2}\right)$ \\
\hline 15 & & & 89 & 10.872 & 0.000 & 94 & 0.628 & 1.003 & $\mathrm{CH}_{2}$ \\
\hline 16 & & & 99 & 0.000 & 1.558 & 111 & 1.907 & 1.210 & $\mathrm{CH}_{2}$ \\
\hline 17 & & & 112 & 8.327 & 0.000 & 112 & 5.846 & 0.260 & $\mathrm{CH}_{2}$ \\
\hline 18 & & & 114 & 7.653 & 0.000 & 118 & 6.499 & 0.067 & $\mathrm{CH}_{2}$ \\
\hline 19 & & & 134 & 15.689 & 0.000 & 131 & 5.472 & 0.328 & $\omega\left(\mathrm{CH}_{3}\right)$ \\
\hline 20 & & & 138 & 0.000 & 0.272 & 139 & 17.142 & 0.208 & $\omega\left[\left(\mathrm{CH}_{3}\right)+\mathrm{NH}_{3}\right]$ \\
\hline 21 & & & 182 & 0.000 & 0.315 & 167 & 1.166 & 0.568 & $\omega\left[\left(\mathrm{CH}_{3}\right)+\mathrm{NH}_{3}\right]$ \\
\hline
\end{tabular}


Optimized Geometry, Charge Distributions, Frontier Molecular Orbital Analysis and NLO Efficiency of BIS (4-Methoxyanilinium) Adipate by Quantum Chemical Calculations

\begin{tabular}{|c|c|c|c|c|c|c|c|c|c|}
\hline \multirow{2}{*}{$\begin{array}{l}\text { Mode } \\
\text { Number }\end{array}$} & \multicolumn{2}{|c|}{ Experimental } & \multicolumn{3}{|c|}{$\mathrm{HF} / 6-311++\mathrm{G}(\mathrm{d}, \mathrm{p})$} & \multicolumn{3}{|c|}{ B3LYP/6-311++G(d,p) } & \multirow{2}{*}{ Assignment } \\
\hline & FT-IR & Raman & $v_{\text {cal }}$ & ${ }^{\mathrm{a}} \mathrm{I}^{\mathrm{IR}}$ & ${ }^{b} I^{\text {Raman }}$ & $v_{\text {cal }}$ & ${ }^{\mathrm{a}} \mathrm{I}{ }^{\mathrm{IR}}$ & ${ }^{\mathrm{b}} \mathrm{I}$ Raman & \\
\hline 22 & & & 194 & 17.858 & 0.000 & 201 & 41.528 & 1.642 & $\mathrm{t}\left(\mathrm{CH}_{3}\right)$ \\
\hline 23 & & & 208 & 0.000 & 4.753 & 219 & 6.370 & 4.839 & $\mathrm{t}\left(\mathrm{CH}_{3}\right)$ \\
\hline 24 & & & 244 & 9.146 & 0.000 & 230 & 4.060 & 0.741 & $\mathrm{t}\left(\mathrm{CH}_{3}\right)$ \\
\hline 25 & & & 244 & 0.000 & 0.817 & 232 & 2.624 & 1.107 & $\omega\left[\left(\mathrm{CH}_{3}\right)+\mathrm{NH}_{3}\right]$ \\
\hline 26 & & & 270 & 0.002 & 0.483 & 247 & 6.051 & 0.350 & $\mathrm{t}\left(\mathrm{CH}_{3}\right)$ \\
\hline 27 & & & 270 & 1.191 & 0.001 & 249 & 1.459 & 0.360 & $\mathrm{t}\left(\mathrm{CH}_{3}\right)$ \\
\hline 28 & & & 339 & 0.000 & 6.554 & 318 & 0.109 & 4.549 & $\mathrm{C}-\mathrm{C}$ \\
\hline 29 & & & 347 & 20.339 & 0.000 & 339 & 22.406 & 0.395 & $\omega\left(\mathrm{NH}_{3}\right)$ \\
\hline 30 & & & 357 & 0.000 & 2.011 & 352 & 8.204 & 4.904 & $\omega\left(\mathrm{NH}_{3}\right)$ \\
\hline 31 & & & 373 & 4.900 & 0.000 & 355 & 3.068 & 0.915 & $\omega\left(\mathrm{NH}_{3}\right)$ \\
\hline 32 & & & 404 & 5.530 & 0.001 & 374 & 3.324 & 3.044 & $\omega\left(\mathrm{NH}_{3}\right)+\gamma(\mathrm{CH})$ \\
\hline 33 & & & 404 & 0.001 & 5.149 & 376 & 1.925 & 1.961 & $\omega\left(\mathrm{NH}_{3}\right)+\gamma(\mathrm{CH})$ \\
\hline 34 & & & 426 & 0.000 & 3.077 & 417 & 0.172 & 2.061 & $\gamma(\mathrm{CH})+\left(\mathrm{NH}_{3}\right)$ \\
\hline 35 & & & 428 & 17.392 & 0.000 & 418 & 4.609 & 0.682 & $\gamma(\mathrm{CH})$ \\
\hline 36 & & & 463 & 0.000 & 5.304 & 429 & 0.376 & 2.620 & $\gamma(\mathrm{CH})$ \\
\hline 37 & & & 463 & 2.651 & 0.000 & 429 & 0.890 & 2.605 & $\mathrm{t}\left(\mathrm{NH}_{3}\right)+(\mathrm{CH})$ \\
\hline 38 & & & 467 & 0.000 & 1.891 & 438 & 2.480 & 1.497 & $\mathrm{t}\left(\mathrm{NH}_{3}\right)+(\mathrm{CH})$ \\
\hline 39 & & & 468 & 15.393 & 0.000 & 445 & 41.174 & 0.603 & $\omega\left(\mathrm{NH}_{3}\right)$ \\
\hline 40 & & & 487 & 19.421 & 0.000 & 473 & 8.356 & 0.445 & $\mathrm{t}\left(\mathrm{NH}_{3}\right)$ \\
\hline 41 & 513 & & 489 & 0.000 & 0.802 & 475 & 7.597 & 0.529 & $\mathrm{t}\left(\mathrm{NH}_{3}\right)$ \\
\hline 42 & & & 569 & 0.570 & 3.081 & 525 & 38.728 & 0.572 & $\mathrm{t}\left(\mathrm{NH}_{3}\right)$ \\
\hline 43 & & & 569 & 5.809 & 0.302 & 526 & 16.399 & 1.184 & $\gamma(\mathrm{CH})$ \\
\hline 44 & & & 580 & 69.983 & 0.000 & 536 & 4.442 & 1.229 & $\gamma(\mathrm{CH})$ \\
\hline 45 & & & 580 & 0.000 & 0.726 & 536 & 5.449 & 1.175 & $\beta(\mathrm{CH})+\rho\left(\mathrm{CH}_{3}\right)$ \\
\hline 46 & 621 & & 663 & 70.342 & 0.000 & 620 & 22.129 & 0.336 & $\beta(\mathrm{CH})+\rho\left(\mathrm{CH}_{3}\right)$ \\
\hline 47 & & & 695 & 0.000 & 4.309 & 651 & 2.742 & 7.484 & $\mathrm{C}=\mathrm{O}$ Vibration \\
\hline 48 & & & 701 & 0.636 & 0.000 & 655 & 0.227 & 6.484 & $\rho\left(\mathrm{CH}_{2}\right)$ \\
\hline 49 & & & 701 & 0.000 & 12.489 & 656 & 1.054 & 4.551 & $\beta(\mathrm{CH})+$ Ring deformation \\
\hline 50 & 117 & 751 & 777 & 0.000 & 2.534 & 717 & 23.932 & 5.343 & $\beta(\mathrm{CH})+$ Ring deformation \\
\hline 51 & & & 779 & 135.974 & 0.000 & 720 & 12.365 & 1.935 & $\gamma(\mathrm{CH})+\left(\mathrm{NH}_{3}\right)$ \\
\hline 52 & & & 787 & 0.000 & 4.919 & 723 & 2.747 & 3.499 & $\gamma(\mathrm{CH})+\left(\mathrm{NH}_{3}\right)$ \\
\hline
\end{tabular}


International Journal of Engineering and Advanced Technology (IJEAT) ISSN: 2249 - 8958, Volume-9, Issue-1S4, December 2019

\begin{tabular}{|c|c|c|c|c|c|c|c|c|c|}
\hline \multirow{2}{*}{$\begin{array}{l}\text { Mode } \\
\text { Number }\end{array}$} & \multicolumn{2}{|c|}{ Experimental } & \multicolumn{3}{|c|}{$\mathrm{HF} / 6-311++\mathrm{G}(\mathrm{d}, \mathrm{p})$} & \multicolumn{3}{|c|}{ B3LYP/6-311++G(d,p) } & \multirow{2}{*}{ Assignment } \\
\hline & FT-IR & Raman & $v_{\text {cal }}$ & ${ }^{\mathrm{a}} \mathrm{I}^{\mathrm{IR}}$ & ${ }^{b} I^{\text {Raman }}$ & $v_{\mathrm{cal}}$ & ${ }^{\mathrm{a}} \mathrm{I}^{\mathrm{IR}}$ & ${ }^{b} I^{\text {Raman }}$ & \\
\hline 53 & & & 787 & 55.744 & 0.000 & 736 & 97.797 & 2.265 & $\gamma(\mathrm{CH})$ \\
\hline 54 & 781 & & 788 & 0.000 & 3.444 & 736 & 76.996 & 1.395 & $v(\mathrm{C}=\mathrm{N}+\mathrm{C}=\mathrm{O})$ \\
\hline 55 & & & 792 & 0.936 & 0.000 & 746 & 2.790 & 1.311 & $v \mathrm{C}-\mathrm{N}+$ Ring breath \\
\hline 56 & & & 818 & 19.527 & 0.000 & 751 & 7.940 & 0.620 & $(\mathrm{C}-\mathrm{H})$ \\
\hline 57 & & & 857 & 0.000 & 1.714 & 803 & 1.392 & 0.181 & $(\mathrm{C}-\mathrm{H})$ \\
\hline 58 & & & 882 & 30.791 & 0.001 & 808 & 3.833 & 0.994 & $\gamma(\mathrm{CH})$ \\
\hline 59 & & & 884 & 0.003 & 9.796 & 810 & 7.023 & 1.392 & $\gamma(\mathrm{CH})$ \\
\hline 60 & & & 906 & 1.990 & 0.027 & 823 & 48.722 & 3.324 & $\gamma(\mathrm{H})+\left(\mathrm{NH}_{3}\right)$ \\
\hline 61 & & & 906 & 0.001 & 75.846 & 824 & 38.482 & 3.277 & $\rho\left(\mathrm{H}_{2}\right)+(\mathrm{H})$ \\
\hline 62 & & & 915 & 1.297 & 0.000 & 844 & 72.960 & 5.907 & $\mathrm{C}-\mathrm{H}+$ Ring breath \\
\hline 63 & & & 915 & 0.000 & 1.744 & 844 & 7.509 & 59.577 & C-H +Ring breath \\
\hline 64 & & & 948 & 184.126 & 0.000 & 883 & 466.583 & 22.205 & $\rho\left(\mathrm{NH}_{3}\right)$ \\
\hline 65 & & 947 & 949 & 0.022 & 1.076 & 886 & 158.098 & 41.891 & $\rho\left(\mathrm{NH}_{3}\right)$ \\
\hline 66 & & & 975 & 44.822 & 0.000 & 903 & 31.353 & 1.189 & $v \mathrm{C}-\mathrm{C}$ \\
\hline 67 & & & 978 & 0.001 & 4.774 & 904 & 14.994 & 4.661 & $\mathrm{C}-\mathrm{H}$ \\
\hline 68 & 947 & & 991 & 771.395 & 0.006 & 929 & 2.461 & 1.171 & $\mathrm{C}-\mathrm{H}$ \\
\hline 69 & & & 993 & 0.221 & 19.909 & 934 & 46.091 & 2.189 & $\gamma(\mathrm{CH})+\left(\mathrm{NH}_{3}\right)$ \\
\hline 70 & & & 1010 & 71.800 & 0.000 & 936 & 33.831 & 3.201 & $\gamma(\mathrm{CH})$ \\
\hline 71 & & & 1049 & 40.681 & 0.001 & 953 & 0.139 & 0.030 & $\gamma(\mathrm{CH})$ \\
\hline 72 & & & 1049 & 0.022 & 1.524 & 954 & 0.267 & 0.037 & $\gamma(\mathrm{CH})$ \\
\hline 73 & & & 1077 & 0.095 & 0.180 & 1013 & 67.170 & 5.939 & $(\mathrm{C}-\mathrm{C})+(\mathrm{O}-\mathrm{H})$ \\
\hline 74 & & & 1077 & 0.708 & 0.024 & 1018 & 76.956 & 8.264 & $\rho(\mathrm{OH})$ \\
\hline 75 & & & 1086 & 1.795 & 0.000 & 1020 & 82.708 & 11.123 & $\beta(\mathrm{O}-\mathrm{H})$ \\
\hline 76 & & & 1098 & 8.478 & 0.001 & 1025 & 27.417 & 1.865 & $\beta(\mathrm{CH})+(\mathrm{O}-\mathrm{H})$ \\
\hline 77 & 1033 & & 1098 & 0.017 & 0.523 & 1025 & 43.545 & 6.374 & $\beta(\mathrm{CH})+(\mathrm{O}-\mathrm{H})$ \\
\hline 78 & & & 1122 & 0.000 & 9.431 & 1053 & 2.931 & 4.929 & $\mathrm{O}-\mathrm{H}+\beta(\mathrm{CH})$ \\
\hline 79 & & & 1148 & 0.000 & 29.224 & 1063 & 66.419 & 15.085 & $\mathrm{C}-\mathrm{O}+\beta(\mathrm{CH})$ \\
\hline 80 & 1152 & & 1160 & 53.116 & 1.585 & 1063 & 18.039 & 6.113 & $\beta(\mathrm{CH})$ \\
\hline 81 & & & 1160 & 43.999 & 1.912 & 1064 & 35.678 & 16.137 & $\rho\left(\mathrm{CH}_{2}\right)$ \\
\hline 82 & & & 1179 & 67.927 & 0.362 & 1088 & 43.372 & 1.749 & $\omega\left(\mathrm{CH}_{2}\right)$ \\
\hline
\end{tabular}


Optimized Geometry, Charge Distributions, Frontier Molecular Orbital Analysis and NLO Efficiency of BIS (4-Methoxyanilinium) Adipate by Quantum Chemical Calculations

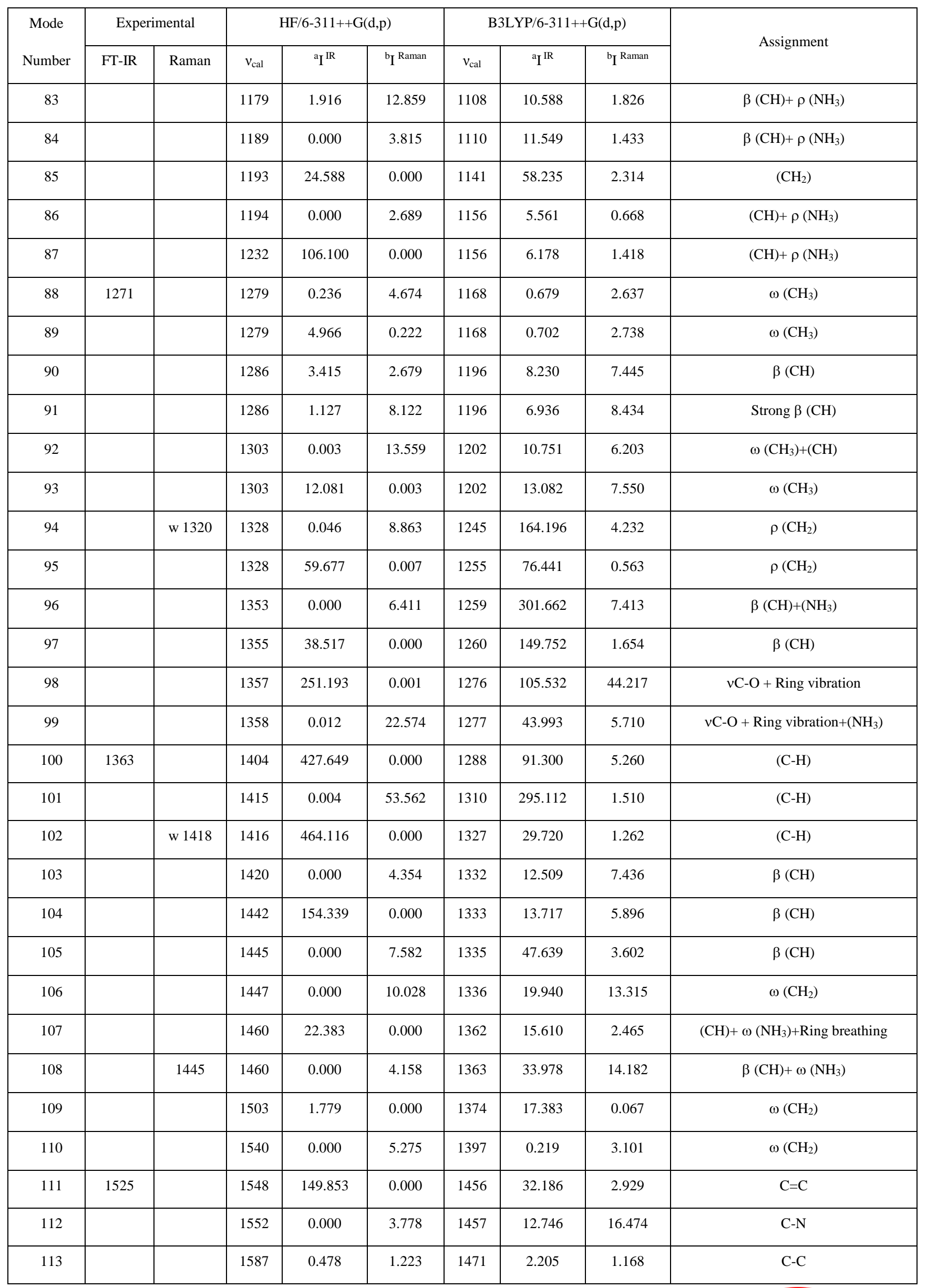


International Journal of Engineering and Advanced Technology (IJEAT) ISSN: 2249 - 8958, Volume-9, Issue-1S4, December 2019

\begin{tabular}{|c|c|c|c|c|c|c|c|c|c|}
\hline \multirow{2}{*}{$\begin{array}{l}\text { Mode } \\
\text { Number }\end{array}$} & \multicolumn{2}{|c|}{ Experimental } & \multicolumn{3}{|c|}{$\mathrm{HF} / 6-311++\mathrm{G}(\mathrm{d}, \mathrm{p})$} & \multicolumn{3}{|c|}{ B3LYP/6-311++G(d,p) } & \multirow{2}{*}{ Assignment } \\
\hline & FT-IR & Raman & $v_{\text {cal }}$ & ${ }^{\mathrm{a}} \mathrm{I}{ }^{\mathrm{IR}}$ & ${ }^{b} I^{\text {Raman }}$ & $v_{\text {cal }}$ & ${ }^{\mathrm{a}} \mathrm{I}^{\mathrm{IR}}$ & ${ }^{b} I^{\text {Raman }}$ & \\
\hline 114 & 1593 & & 1587 & 0.450 & 1.299 & 1471 & 1.981 & 1.666 & $\omega(\mathrm{CH} 3)+\beta(\mathrm{CH})+\omega(\mathrm{NH} 3)$ \\
\hline 115 & & & 1603 & 21.928 & 0.000 & 1476 & 11.669 & 2.908 & $\beta(\mathrm{CH})+\rho\left(\mathrm{NH}_{3}\right)+$ Ring breathing \\
\hline 116 & & & 1605 & 44.358 & 0.001 & 1476 & 9.470 & 3.173 & $\omega\left(\mathrm{CH}_{3}\right)$ \\
\hline 117 & & & 1605 & 0.007 & 5.419 & 1478 & 10.746 & 0.372 & $\omega\left(\mathrm{CH}_{3}\right)$ \\
\hline 118 & & & 1607 & 0.000 & 28.806 & 1482 & 3.339 & 35.459 & $\omega\left(\mathrm{CH}_{2}\right)$ \\
\hline 119 & & & 1617 & 0.000 & 10.077 & 1492 & 8.944 & 13.945 & $\rho\left(\mathrm{CH}_{2}\right)$ \\
\hline 120 & & & 1620 & 4.413 & 17.587 & 1492 & 9.052 & 13.736 & $\rho\left(\mathrm{CH}_{3}\right)$ \\
\hline 121 & & & 1620 & 10.841 & 7.160 & 1495 & 0.174 & 10.993 & $\omega\left(\mathrm{CH}_{3}\right)$ \\
\hline 122 & & & 1630 & 56.895 & 0.003 & 1506 & 39.835 & 4.844 & $\omega\left(\mathrm{CH}_{2}\right)$ \\
\hline 123 & $\mathrm{~m} 1635$ & & 1631 & 0.014 & 11.487 & 1506 & 43.941 & 4.742 & $\delta\left(\mathrm{CH}_{3}\right)$ \\
\hline 124 & & & 1631 & 14.605 & 0.000 & 1512 & 12.766 & 0.050 & $\delta\left(\mathrm{CH}_{3}\right)$ \\
\hline 125 & & & 1676 & 565.120 & 0.000 & 1544 & 230.827 & 2.262 & $(\mathrm{C}-\mathrm{H})+\rho\left(\mathrm{CH}_{2}\right)$ \\
\hline 126 & & & 1676 & 0.003 & 1.091 & 1544 & 289.154 & 1.040 & $\rho\left(\mathrm{NH}_{3}\right)+$ Ring breathing \\
\hline 127 & & & 1776 & 12.214 & 0.069 & 1629 & 5.050 & 7.455 & Ring breathing \\
\hline 128 & & w 2612 & 1776 & 0.054 & 15.743 & 1629 & 4.342 & 7.269 & $\rho\left(\mathrm{NH}_{3}\right)+\mathrm{C}=\mathrm{C}, \mathrm{C}-\mathrm{C}+v \mathrm{C}-\mathrm{H}$ \\
\hline 129 & & & 1798 & 3.893 & 14.276 & 1659 & 19.584 & 51.498 & $\delta\left(\mathrm{NH}_{3}\right)+$ Ring vibration \\
\hline 130 & & & 1798 & 125.368 & 0.444 & 1659 & 18.391 & 50.961 & $\delta\left(\mathrm{NH}_{3}\right)+$ Ring vibration \\
\hline 131 & & & 1817 & 0.012 & 90.301 & 1668 & 32.382 & 37.671 & $\delta\left(\mathrm{NH}_{3}\right)$ \\
\hline 132 & & & 1817 & 17.828 & 0.060 & 1670 & 36.747 & 29.436 & $\delta\left(\mathrm{NH}_{3}\right)$ \\
\hline 133 & 1950 & & 1959 & 923.306 & 0.000 & 1763 & 292.659 & 4.785 & $v \mathrm{C}=\mathrm{O}+\omega(\mathrm{OH})$ \\
\hline 134 & w2349 & s 2816 & 1962 & 0.001 & 17.331 & 1767 & 370.950 & 33.482 & $v \mathrm{C}=\mathrm{O}+\omega(\mathrm{OH})$ \\
\hline 135 & 2760 & s 2919 & 3158 & 0.000 & 141.895 & 2999 & 51.195 & 134.252 & $\gamma \mathrm{O}-\mathrm{H}$ \\
\hline 136 & 2864 & & 3159 & 34.562 & 195.989 & 2999 & 68.753 & 210.806 & $v_{\mathrm{s}} \mathrm{CH}_{3}$ \\
\hline 137 & & & 3159 & 74.886 & 90.357 & 3017 & 3.666 & 209.255 & $v_{\text {as }} \mathrm{CH}_{2}$ \\
\hline 138 & & & 3166 & 36.716 & 0.000 & 3021 & 16.272 & 34.428 & $v_{\mathrm{as}} \mathrm{CH}_{2}$ \\
\hline 139 & 2987 & s3024 & 3192 & 0.009 & 109.860 & 3040 & 3.148 & 97.353 & N...O-H \\
\hline 140 & & & 3195 & 46.340 & 0.018 & 3043 & 35.288 & 10.835 & $v \mathrm{C}-\mathrm{H}$ \\
\hline 141 & & & 3201 & 0.001 & 132.670 & 3054 & 3.251 & 80.730 & $\mathrm{vC}-\mathrm{H}$ \\
\hline 142 & & s2923 & 3216 & 16.925 & 69.247 & 3056 & 41.208 & 61.295 & $v_{\mathrm{as}} \mathrm{CH}_{2}$ \\
\hline 143 & & & 3216 & 87.985 & 13.320 & 3056 & 40.453 & 58.026 & $v_{\mathrm{as}} \mathrm{CH}_{2}$ of $\mathrm{CH}_{3}$ \\
\hline
\end{tabular}


Optimized Geometry, Charge Distributions, Frontier Molecular Orbital Analysis and NLO Efficiency of BIS (4-Methoxyanilinium) Adipate by Quantum Chemical Calculations

\begin{tabular}{|c|c|c|c|c|c|c|c|c|c|}
\hline \multirow{2}{*}{$\begin{array}{l}\text { Mode } \\
\text { Number }\end{array}$} & \multicolumn{2}{|c|}{ Experimental } & \multicolumn{3}{|c|}{$\mathrm{HF} / 6-311++\mathrm{G}(\mathrm{d}, \mathrm{p})$} & \multicolumn{3}{|c|}{ B3LYP/6-311++G(d,p) } & \multirow{2}{*}{ Assignment } \\
\hline & FT-IR & Raman & $v_{\text {cal }}$ & ${ }^{\mathrm{a}} \mathrm{I}{ }^{\mathrm{IR}}$ & ${ }^{\mathrm{b}} \mathrm{I}^{\text {Raman }}$ & $v_{\text {cal }}$ & ${ }^{\mathrm{a}} \mathrm{I}{ }^{\mathrm{IR}}$ & ${ }^{\mathrm{b}} \mathrm{I}$ Raman & \\
\hline 144 & w3132 & & 3219 & 20.287 & 0.000 & 3073 & 14.552 & 28.178 & $v_{\mathrm{as}} \mathrm{CH}_{2}$ \\
\hline 145 & & & 3257 & 0.000 & 58.877 & 3104 & 36.892 & 50.835 & $v \mathrm{C}-\mathrm{H}$ \\
\hline 146 & & & 3261 & 59.232 & 0.000 & 3107 & 41.377 & 22.661 & $v \mathrm{C}-\mathrm{H}$ \\
\hline 147 & & & 3279 & 87.547 & 0.368 & 3131 & 31.170 & 137.568 & $v_{\text {as }} \mathrm{CH}_{3}$ \\
\hline 148 & & & 3279 & 0.121 & 265.626 & 3131 & 29.688 & 132.155 & $v_{\text {as }} \mathrm{CH}_{3}$ \\
\hline 149 & & & 3316 & 4.326 & 118.040 & 3159 & 659.126 & 65.583 & $v_{\text {as }} \mathrm{C}-\mathrm{H}$ \\
\hline 150 & & & 3316 & 28.037 & 18.210 & 3160 & 952.960 & 101.221 & $v(\mathrm{C}-\mathrm{H}+\mathrm{O}-\mathrm{H})$ \\
\hline 151 & 3242 & & 3326 & 13.626 & 30.481 & 3161 & 2616.119 & 107.871 & $v(\mathrm{C}-\mathrm{H}+\mathrm{O}-\mathrm{H})$ \\
\hline 152 & & & 3326 & 4.625 & 89.802 & 3166 & 186.311 & 1199.658 & $v \mathrm{O}-\mathrm{H}$ \\
\hline 153 & & & 3353 & 11.653 & 54.436 & 3167 & 10.025 & 93.623 & $v \mathrm{O}-\mathrm{H}$ \\
\hline 154 & & & 3353 & 3.186 & 199.023 & 3167 & 12.584 & 95.507 & $v \mathrm{C}-\mathrm{H}$ \\
\hline 155 & $3255 w$ & & 3366 & 4.204 & 121.356 & 3193 & 2.483 & 160.586 & $v \mathrm{C}-\mathrm{H}$ \\
\hline 156 & & & 3366 & 12.765 & 39.969 & 3193 & 2.415 & 160.045 & $v \mathrm{C}-\mathrm{H}$ \\
\hline 157 & w3323 & & 3740 & 21.741 & 117.941 & 3204 & 5.737 & 88.618 & $v \mathrm{C}=\mathrm{H}$ \\
\hline 158 & & & 3740 & 16.538 & 155.017 & 3205 & 5.607 & 92.225 & $v \mathrm{~N}-\mathrm{H}$ \\
\hline 159 & & & 3823 & 2192.191 & 0.132 & 3486 & 71.280 & 163.555 & $v_{\mathrm{s}}\left(\mathrm{NH}_{3}\right)$ \\
\hline 160 & & & 3825 & 0.574 & 491.344 & 3487 & 62.528 & 182.745 & $v_{\text {as }}\left(\mathrm{NH}_{3}\right)$ \\
\hline 161 & & & 3831 & 191.489 & 2.756 & 3585 & 30.563 & 60.052 & $v_{\text {as }}\left(\mathrm{NH}_{3}\right)$ \\
\hline 162 & & & 3831 & 5.307 & 101.720 & 3585 & 29.324 & 66.855 & $v_{\text {as }}\left(\mathrm{NH}_{3}\right)$ \\
\hline
\end{tabular}

Generally, the $\mathrm{N}-\mathrm{H}$ stretching vibrations occur in the region of $3600-3300 \mathrm{~cm}^{-1}$ in amines. In the present study, $\mathrm{N}-\mathrm{H}$ symmetric stretching mode is assigned at $3888 \mathrm{~cm}^{-1}$ in FT-IR and inactive in Raman spectrum. In this compound, each of the $\mathrm{N}-\mathrm{H}$ bond of the $\left(\mathrm{NH}_{3}\right)^{+}$group is hydrogen bonded with the oxygen atom of the adipiate anion. In 4MAA an intense peak calculated at 3831 and $3585 \mathrm{~cm}^{-1}$ in $\mathrm{HF}$ and DFT/B3LYP methods respectively, are assigned to $\mathrm{NH}_{3}{ }^{+}$ asymmetric mode. The rocking vibration of $\mathrm{NH}_{3}{ }^{+}$group is observed at 1676 and $1544 \mathrm{~cm}^{-1}$ in HF and DFT/B3LYP calculations respectively whereas the corresponding experimental IR and Raman bands were absent. The $\mathrm{NH}_{3}{ }^{+}$ wagging vibration is observed in IR at $1593 \mathrm{~cm}^{-1}$ and in Raman spectrum it is absent.

\section{Vibrations of N-H...O}

The intermolecular hydrogen bond was formed between amine $\mathrm{N}$ atom and carbonyl $\mathrm{O}$ atom after optimization. The present structure has $\mathrm{N}-\mathrm{H}$...O bond of cation, which is observed as medium peak at $3024 \mathrm{~cm}^{-1}$ in Raman spectrum and inactive in IR spectrum. This band is theoretically calculated at $3192 \mathrm{~cm}^{-1}$ and $3040 \mathrm{~cm}^{-1} \mathrm{HF}$ and B3LYP methods, respectively. The stretching vibration of this $\mathrm{N}-\mathrm{H}$... O hydrogen bond interactions is proved and it is good correlation with the theoretical calculations.

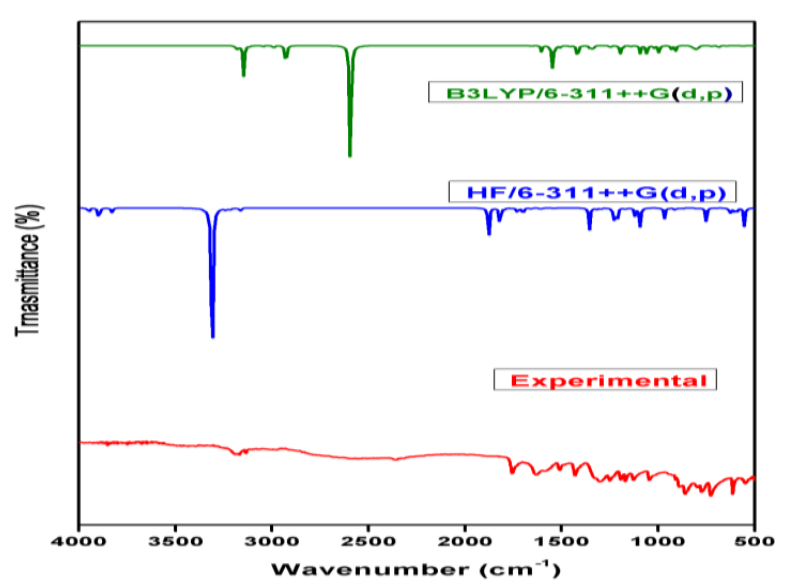

Fig. 6. FT-IR spectra for 4MAA 


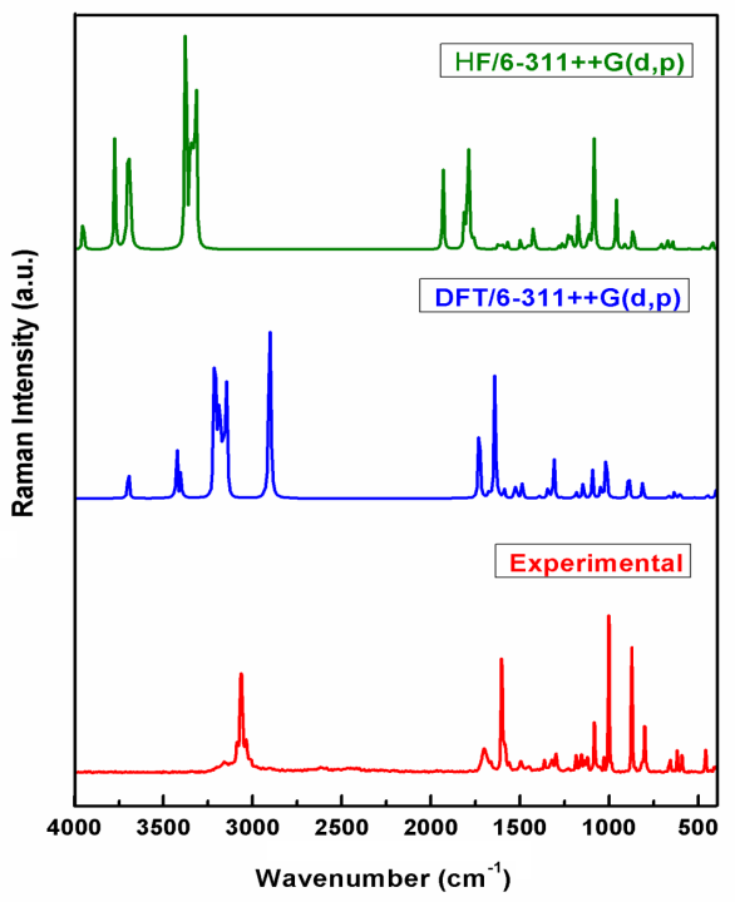

\section{Nonlinear optical behaviour}

Nonlinear optics is at the forefront of current research because of its significance in provided that the key functions of frequency conversion, optical limiter and optical data storage devices for the emerging technologies. Non linear optical parameters such as dipole moment $(\mu)$, polarizability $(\alpha)$, first-order hyperpolarizability $(\beta)$ and second order hyperpolarizability $(\gamma)$ were calculated at the B3LYP methods with $6-311++\mathrm{G}(\mathrm{d}, \mathrm{p})$ levels and the calculated values are reported in Table IV. Therefore, the second order hyperpolarizability calculation [9-11] was attempted and the second order hyperpolarizability value is comparably 74 times greater than the urea $(4.728 \times 10-36$ esu). These above results show that the present molecule is excellent material for optoelectronic applications

Fig. 7. FT-Raman spectra for 4MAA

Table IV: Non linear optical parameters of 4MAA calculated by DFT/B3LYP with 6-311++G(d,p) levels

\begin{tabular}{|c|c|c|c|c|c|c|c|c|}
\hline Parameter & a.u & $\begin{array}{c}\text { esu } \\
\left(\times 10^{-24}\right)\end{array}$ & Parameter & a.u & $\begin{array}{c}\text { esu } \\
\left(\times 10^{-33}\right)\end{array}$ & Parameter & a.u & $\begin{array}{c}\text { esu } \\
\left(10^{-36}\right)\end{array}$ \\
\hline$\mu_{\mathrm{x}}$ & -0.0102 & & $\beta_{\mathrm{xxx}}$ & -0.0375 & -0.33146 & $\gamma_{\mathrm{xxxx}}$ & 282531.9 & 262.469 \\
\hline$\mu_{\mathrm{y}}$ & 0.0040 & & $\beta_{\mathrm{xxy}}$ & -0.0054 & -0.04773 & $\gamma_{\mathrm{xxyy}}$ & 99379.29 & 114.323 \\
\hline$\mu_{\mathrm{z}}$ & 0.0024 & & $\beta_{\mathrm{xyy}}$ & -0.0202 & -0.17855 & $\gamma_{\mathrm{yyyy}}$ & 586289 & 101.346 \\
\hline$\mu$ & 0.0112 & & $\beta_{\mathrm{yyy}}$ & -0.0002 & -0.00177 & $\gamma_{\mathrm{yyzz}}$ & 582984.2 & 237.619 \\
\hline$\alpha_{\mathrm{xx}}$ & -0.7528 & -0.11156 & $\beta_{\mathrm{xxz}}$ & 0.0166 & 0.146727 & $\gamma_{\mathrm{xxzz}}$ & 65192.57 & 128.441 \\
\hline$\alpha_{\mathrm{xy}}$ & -27.6109 & -4.09194 & $\beta_{\mathrm{xyz}}$ & 0.0073 & 0.064525 & $\gamma_{\mathrm{zzzz}}$ & 145979.6 & 441.0920 \\
\hline$\alpha_{\mathrm{yy}}$ & -125.0021 & -18.5253 & $\beta_{\mathrm{yyz}}$ & -0.0015 & -0.01326 & $\gamma$ & 501983 & 353.135 \\
\hline$\alpha_{\mathrm{xz}}$ & 30.9564 & 4.587738 & $\beta_{\mathrm{xzz}}$ & -0.0027 & -0.02387 & & & \\
\hline$\alpha_{\mathrm{yz}}$ & -30.5133 & -4.52207 & $\beta_{\mathrm{yzz}}$ & -0.0015 & -0.01326 & & & \\
\hline$\alpha_{z z}$ & -179.8625 & -26.6556 & $\beta_{z z z}$ & 0.0023 & 0.02033 & & & \\
\hline$\alpha$ & -101.8724 & -15.0974 & $\beta_{0}$ & 0.06326 & 0.05591 & & & \\
\hline$\Delta \alpha$ & 356.8715 & 52.8883 & & & & & & \\
\hline
\end{tabular}




\section{Optimized Geometry, Charge Distributions, Frontier Molecular Orbital Analysis and NLO Efficiency of BIS (4-Methoxyanilinium) Adipate by Quantum Chemical Calculations}

\section{CONCLUSION}

The optimized geometrical parameters, NLO properties, electronic properties and vibrational assignments of the 4MAA have been studied by HF and DFT calculations. The complete theoretical and experimental vibrational assignments were analyzed as well as compared. The good agreement of the calculated and observed vibrational spectra reveals the advantages of higher basis sets for computational calculations. The FMOs energies show that charge transfer occurs within the molecule. The title compounds exhibited good NLO property and were much 74 times hgher than that of reference urea.

\section{REFERENCES}

[1] M. Suresh, S. Asath Bahadur, and S. Athimoolam, Synthesis, Growth, structural, spectral, thermal and microhardness studies of a new hydrogen bonded organic nonlinear optical material: L-Valinium $\mathrm{p}$ -toluenesulfonate monohydrate (LVPT), J. Mol. Struct., 1112 , 2016,pp. 71-80.

[2] P. Karthiga Devi, K. Venkatachalam, Growth, spectroscopic, mechanical, thermal, antimicrobial and DFT studies of piperidinium hydrogen oxalateJ. Mater Sci: Mater Electron. Mat. Sci.: Mat. Electr.,27(8),2016,pp.8590-8598.

[3] N. Bhuvaneswari, N. Priyadharsini, S. Sivakumar, K. Venkatachalam, V. Siva, Growth, vibrational, optical, thermal, magnetic and dielectric behavior of organo-metallic tetramethyl ammonium cadmium chloride crystal, J Therm. Anal. Calorim.136, 2018. pp.411-417.

[4] F.A. Diaz, C.O. Sanchez, M.A. Del Valle, J.L. Torres, L.H. Tagle, Synth. Met. (2001) 25-31

[5] S.C. Ng, L. Xu, Adv. Mater. 10, 1998, pp. 1525-1530.

[6] V. Siva, A. Shameem, A. Murugan, S. Athimoolam, M. Suresh, S Asath Bahadur, Supramolecular architecture, hydrogen bonding assembly and Hirshfeld surface analysis of charge transfer adipate salt of 4-methoxyaniline, Chem. data collect., 24, 2019,pp. 100281.

[7] M.J. Frisch et al, Gaussian Inc., Wallingford, CT, 2009.

[8] R. Dennington, T. Keith, J. Millam. Gauss View, Version 5.0.8 Semichem Inc., Shawnee Mission KS. 2013.

[9] V. Siva, A. Shameem, S. Suresh Kumar, M. Raja, S. Athimoolam, S Asath Bahadur. Structural, spectral, Quantum chemical and thermal studies on a new NLO crystal: Guanidinium Cinnamate, J Mat. Sci: Mat. Electr. 28(17), 2017,pp. 12484-12496.

[10] S. Thangarasu, V. Siva, S. Athimoolam, S. Asath Bahadur, Molecular structure, spectroscopic and quantum chemical studies on benzoic acid and succinic acid co-crystals of 2-Aminopyrimidine, J Theo. Comp. Chem., 174 (2018) 1850021.

[11] V. Siva, S. Suresh Kumar, M. Suresh, M. Raja, S. Athimoolam, S. Asath Bahadur. N-H...O hydrogen bonded novel nonlinear optical semiorganic crystal (4-methoxyanilinium trifluoroacetate) studied through theoretical and experimental methods, J. Mol. Struct., 1133, 2017,pp.163-171.

\section{AUTHORS PROFILE}

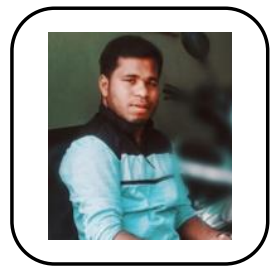

Dr. V. Siva obtained Ph.D in Physics from Kalasalingam Academy of Research and Education in 2018. He has received his Bachelor's Degree and Master's Degree in Physics from Government Arts College (Autonomous), Coimbatore, which is affiliated to Bharathiar University in the years 2010 and 2012, respectively. He has expertise in the areas of Crystallography, Crystal growth and Computational Physics. To his credit, he has published more than 20 articles in journals of international repute besides ten more as proceedings. Further, he has presented papers in various conferences and seminars. His current research interest is development of Metal-Organic Frameworks (MOFs) for sensor and energy storage application.

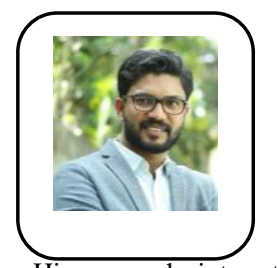

A. Shameem is a Research scholar in the Department of Physics, School of Advanced Sciences at Kalasalingam Academy of Research and Education, India. He received his B.Sc. degree in Physics from Jamal MohamedCollege, Tiruchirappalli, in 2010, and his M.Sc. degree in Physics from Government Arts College (Autonomous), Coimbatore, in 2012 His research interest is in the field of nanomaterials for energy and environmental applications.

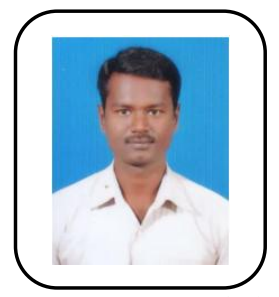

Anbazhagan Murugan is a Senior Research Fellow in the Department of Physics at Kalasalingam Academy of Research and Education. He has joined as Junior Research Fellow in the Department of Physics under DST-SERB funded project, Govt. of India in the year of 2017. He has received his Master's Degree in Physics from Government Arts College (Autonomous), Coimbatore, which is affiliated to Bharathiar University in the year 2013.His research focus is thin film for solar cell applicationsand the design of materials enhanced for energy applications.

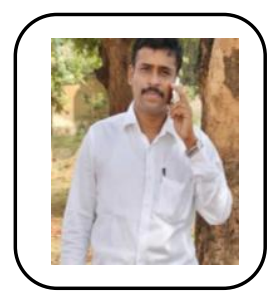

Dr. Shunmuganarayanan Athimoolam, Head of the Department in Physics at University College of Engineering Nagercoil, Anna University Constituent College, India. He has received his Doctoral degree in the area of X-ray Crystallography from Madurai Kamaraj University in the year 2008. He joined as Assistant Professor of Physics at University College of Engineering Nagercoil from the year 2009. His current research interests are Crystal and molecular structure analyses, hydrogen bonding analyses, Coordination and inclusion compounds, IR and Raman spectral analyses, DFT related theoretical studies, nano-drug synthesis and characterization, etc. He has published more than 150 papers in international/national journals in addition to extensive presentations in conferences.

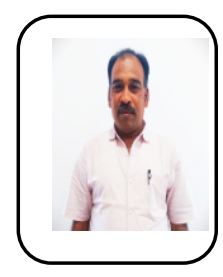

Dr. S. Asath Bahadur is a Senior Professor in the Department of Physics, School of Advanced Sciences at Kalasalingam Academy of Research and Education, India. He has obtained Ph.D in the area of X-ray crystallography from Madurai Kamaraj University in 1994 and also received his M.Phil and M.Sc. Degree in physics from Madurai Kamaraj University, $\mathrm{He}$ has expertise in the areas of Crystallography and Material science. He has published more than eighty five peer review research articles in journals of international repute besides seventy more as proceedings. 